- Case Report

\title{
Angle closure glaucoma in contralateral eye induced by topical atropine used for treatment of corneal ulcer in the fellow eye
}

\author{
S Shah, P Lavaju \\ Department of Ophthalmology, BP Koirala Institute of Health Sciences, Dharan, Nepal
}

\begin{abstract}
Acute angle closure glaucoma (AACG) can be induced by topical mydriatics which are used as cycloplegic and to prevent synechiae while treating corneal ulcer disease. Development of AACG in opposite eye by use of topical mydriatic in one eye is a very rare condition. We report a very rare case of a 45 -year-old male who while using topical atropine in the right eye for corneal ulcer developed atropine induced AACG in the left. The AACG in this patient was managed by medications and laser iridotomy followed by trabeculectomy. The aim of this case report is to raise the awareness about this rare adverse effect of topical use of atropine where AACG can be induced in contralateral eye which warrants the clinician to be more careful and vigilant while prescribing atropine so that timely intervention can prevent the devastating condition leading to blindness.
\end{abstract}

Keywords: acute angle closure glaucoma, atropine, mydriatic

\section{Introduction}

One of the most important causes of acute angle closure glaucoma (AACG) is the application of either systemic or topical medications and the eyes with reduced anterior chamber depth, thicker lens and steeper corneal curvature are at increased risk. ${ }^{1,2}$ Atropine acts as a mydriatic and may become an initiating factor for AACG. To our knowledge, it has not been reported that atropine when used in one eye, can induce acute congestive glaucoma in the contralateral eye. We report a rare case of AACG in the contralateral eye induced by topical atropine used in one eye which was successfully managed with trabeculectomy.

\section{Case report}

A 45- year- old male presented to out patient department of ophthalmology with pain, redness and diminution of vision of right eye associated with whitish discoloration of black part of the eye for one and a half months and pain, redness and diminution of vision in the left eye for the last fifteen days.

He was being treated for corneal ulcer in his right eye by a local practitioner.Initially he was prescribed eye drops moxifloxacin and atropine and acyclovir 3\% eye

Address for correspondence: Dr Sangeeta Shah, MD Senior Resident, Department of Ophthalmology, B. P. Koirala Institute of Health Sciences, Dharan, Nepal

E-mail: sangu01@yahoo.com ointment. Since there was no improvement with these drugs he went to another periphery hospital after 10 days and was treated with eye drops chloromphenicol, ofloxacin, fluconazole, atropine, artificial tears in the right eye along with eye ointment acyclovir 3\%. The patient felt symptomatically better in the right eye but by this time he developed redness and pain in the left eye associated with visual diminution for which he came to our hospital.

The patient's visual acuity recorded was perception of light in the right eye and hand movement in the left eye. The projection of rays was inaccurate in the right eye but accurate in the left. The ocular findings of the right eye were ciliary congestion, chemosis, discharge, sloughing corneal ulcer (figure 1) and shallow anterior chamber with fibrinous exudates. The pupil and other inner details were not visible due to hazy media. The ocular findings of the left eye were ciliary congestion, hazy cornea due to corneal edema, shallow anterior chamber of vanHerick grade II with presence of cells +1 . The pupil was round, regular, mid dilated (figure 2) and very sluggish in reaction to light. The lens showed grade I nuclear sclerosis with minimal posterior subcapsular cataract. The vitreous was clear and the fundus examination revealed well defined margin of the optic disc with cup to disc ratio of 0.8 along with 
nasalization of vessels, bayoneting signs, lamellar dot signs and a bright foveal reflex. The intraocular pressure (IOP), measured with applanation tonometer, was 56 $\mathrm{mm}$ of $\mathrm{Hg}$ in the left eye and the right eye was digitally soft. Gonioscopy revealed Shaffer's grade II and peripheral anterior synechiae from 10-11 o'clock positionin the left eye. Ultrasound B-scan of the right eye showed a normal posterior segment.

With the working diagnosis of RE non-healing sloughing corneal ulcer and LE atropine induced AACG, the patient was started on one hourly eye drops of fortified cefazoline $5 \%$ and fortified tobramycin $1.3 \%$, fluconazole $0.3 \%$ two hourly, tropicamide $1 \%$ thrice daily with punctal occlusion in the right eye. The left eye received eye drops prednisolone acetate $1 \%$ six hourly and timolol $0.5 \%$ twice daily. Injection mannitol $20 \%$ was given intravenously stat and tablet acetazolamide $250 \mathrm{mg}$ six hourly.

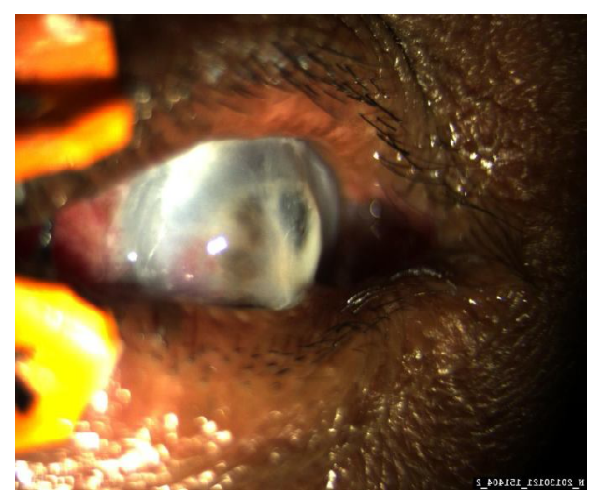

Figure 1: Photograph of the right eye showing sloughing corneal ulcer

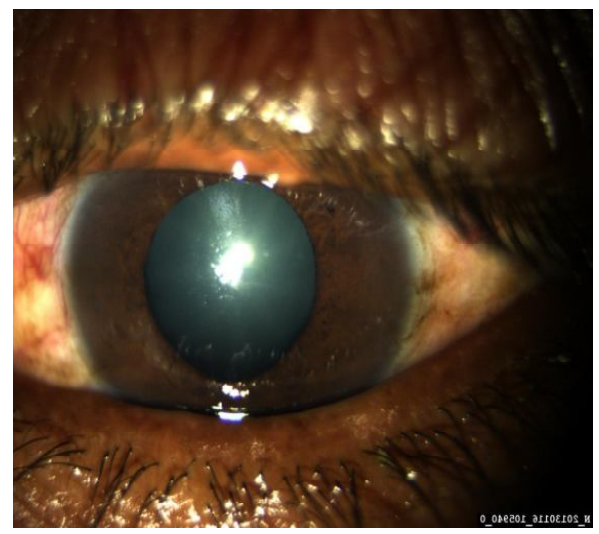

Figure 2: Photograph of the left eye showing mid dilated pupil and shallow $\mathrm{AC}$

$\mathrm{Nd}$ :Yag laser peripheral iridotomy was done in the left eye at 11 o'clock position. Even with this, the IOP was still high therefore eye drops brimonidine twice daily and dorzolamide thrice daily were added. The pressure then dropped to $24 \mathrm{~mm}$ of $\mathrm{Hg}$.

Trabeculectomy with cataract extraction was performed on the left eye ten days after evisceration of the right eye for non-healing sloughing corneal ulcer. Since the right eye showed no improvement of the corneal ulcer with medications and had no chance of regaining useful vision, this step was taken to prevent the spread of infection to the left eye during/after trabeculectomy. As the IOP of the left eye was not controlled by medications and peripheral iridotomy, early trabeculectomy of the left eye was performed to prevent further damage to the optic nerve.

At discharge, the patient had a well controlled IOP of $12 \mathrm{~mm}$ of $\mathrm{Hg}$ in the left eye with improved visual acuity from hand movement at initial presentation to 6/18.

\section{Discussion}

Atropine is a widely used anticholinergic drug for the treatment of corneal ulcer as it acts as a cycloplegic agent relieving pain and a mydriatic agent preventing/ breaking the posterior synechiae. It is often prescribed incases of anterior uveitis, corneal ulcers and in postoperative cases of pediatric cataract. Acute angle closure glaucoma occurs while the pupil constricts over hours following dilation when it is mid dilated to a diameter of 3-4.5 mm. During this phase, the posterior vector force of the iris sphincter muscle reaches its maximum. The peripheral iris is under less tension and is more easily pushed forward into contact with the trabecular meshwork. The dilation may also thicken and bunch the peripheral iris in the angle. ${ }^{3}$

The likely explanation why the right eye did not suffer from acute angle closure glaucoma in our case is that it was under the influence of atropine the whole time. But in the left eye, the attack must have occurred when the mydriatic effect of atropine was wearing off and the pupil began to constrict. The effect of atropine could be due to its systemic absorption through conjunctiva of the right eye and/or the nasal mucosa. ${ }^{4}$ The other possible explanation could be the spill over of the drop from the right eye to the left while changing the position from supine to left lateral.

Topical cycloplegics have been shown to cause significant IOP elevation in $2 \%$ of the apparently normal population, increasing to $23 \%$ of patients with known primary open-angle glaucoma. Berdy et $\mathrm{al}^{4}$ presented 
two patients who developed signs and symptoms of angle closure glaucoma after receiving aerosolized atropine for treatment of chronic obstructive pulmonary disease.

In our case, the patient presented after fifteen days of initiation of the symptoms in the left eye. The actual depth of the anterior chamber prior to the attack had not been documented.Since there was no any history supportive of prior attack of glaucoma, neither any known risk factor for glaucoma in our patient; we presumed the use of topical atropine to be the causative agent of glaucoma in this patient.

\section{Conclusion}

The use of topical atropine sulphate in one eye can lead to acute-angle closure glaucoma in the other eye. Therefore, it should be used carefully and this complication should be kept in mind while examining the patients using topical atropine so that early intervention can prevent this catastrophic event which can result in blindness.

\section{References}

1. George R, Paul PG, Baskaran M, Ramesh S, Raju $\mathrm{P}$, Arvind $\mathrm{H}$, et al. Ocular biometry in occludable angles and angle closure glaucoma: a population based survey. Br J Ophthalmol 2003;87(4):399402.

2. Frideman DS, Gazzrd G, Foster P, Devereux J, Broman A, Quigley $\mathrm{H}$, et al. Ultrasonographicbiomicroscopy, Scheimpflug photography, and novel provocative tests in contralateral eyes of Chinese patients initially seen with acute angle closure. Arch Ophthalmol 2003; 121(5):633-42.

3. Gray RH, Hoare NJ, Ayliffe WHR. Efficacy of NdYag laser iridotomies in acute angle closure glaucoma. Br J Ophthalmol 1989;73(3):182-5.

4. Berdy GJ, Berdy SS, Odin LS, Hirst LW. Angle closure glaucoma precipitated by aerosolized atropine.Arch Intern Med 1991;151(8):1658-60.

5. Portney GL, Purcell TW. The influence of tropicamide on intraocular pressure. Ann Ophthalmol 1975;7(1):31-34. 